\title{
A NOTE ON THE CONTINUOUS EXTENSIONS OF INJECTIVE MORPHISMS BETWEEN FREE GROUPS TO RELATIVELY FREE PROFINITE GROUPS
}

\author{
Thierry Coulbois, Mark SAPIR And Pascal Weil
}

\begin{abstract}
Let $\mathbf{V}$ be a pseudovariety of finite groups such that free groups are residually $\mathbf{V}$, and let $\varphi: F(A) \rightarrow F(B)$ be an injective morphism between finitely generated free groups. We characterize the situations where the continuous extension $\hat{\varphi}$ of $\varphi$ between the pro- $\mathbf{V}$ completions of $F(A)$ and $F(B)$ is also injective. In particular, if $\mathbf{V}$ is extension-closed, this is the case if and only if $\varphi(F(A))$ and its pro- $\mathbf{V}$ closure in $F(B)$ have the same rank. We examine a number of situations where the injectivity of $\hat{\varphi}$ can be asserted, or at least decided, and we draw a few corollaries.
\end{abstract}

In this paper, we are interested in the pro- $\mathbf{V}$ topologies on finitely generated free groups, where $\mathbf{V}$ is a pseudovariety of groups (a class of finite groups closed under taking subgroups, quotients and finite direct products). These topologies were introduced in the 1950 s by Hall. When $\mathbf{V}$ is the class of all finite groups, the finite index subgroups are exactly the open subgroups, and Hall proved [6] that every finitely generated subgroup is closed. More recent papers (Ribes and Zalesskiu [9], Margolis, Sapir and Weil [7], Weil [12]) focused on the problem of effectively computing the pro-V closure $\mathrm{Cl}_{\mathbf{V}}(H)$ of a given finitely generated subgroup $H$ of a free group. It is known for instance that if $\mathbf{V}$ is extension-closed, then $\mathrm{Cl}_{\mathbf{V}}(H)$ has finite rank, at most equal to the rank of $H[\mathbf{9}]$. In general, a finite rank subgroup may have an infinite rank closure (e.g. if $\mathbf{V}$ is the pseudovariety of finite abelian groups), or it may be the case that if $H$ is a finite rank subgroup, then its closure

2000 Mathematics Subject Classification. 20E18, $20 \mathrm{E} 05$.

Key words. Profinite topology, rank of subgroups of free groups, monomorphisms between free groups.

This paper was prepared while the second author was an invited Professor at Université Bordeaux-1. The third author also acknowledges partial support from 1999 INTAS grant number 1224 Combinatorial and geometric theory of groups and semigroups and its applications to computer science. 
always has finite rank, possibly greater than the rank of $H$ (e.g. if $\mathbf{V}$ is the pseudovariety of finite nilpotent groups [7]). It is interesting to note that deciding whether a given subgroup is pro- $\mathbf{V}$-closed is equivalent to deciding an extension property for a certain set of partial isomorphisms of a finite set [ $\mathbf{7}]$. If $\mathbf{V}$ consists of all finite $p$-groups, for some fixed prime $p$, the closure of a given finite rank subgroup can be effectively computed [9], in polynomial time [7]. On the other hand, it is not known whether the pro-solvable closure of a finite rank subgroup is effectively computable; a positive solution for this difficult open question would have interesting consequences in finite monoid theory [7] and in computational complexity (Straubing and Thérien [10]).

It is also known that finite rank closed subgroups are free factors of clopen subgroups, and that the converse holds if $\mathbf{V}$ is extensionclosed [9]. Moreover, again in the extension-closed case, if $H$ is a finite rank pro-V-closed subgroup of a free group $F$, then the pro- $\mathbf{V}$ topology of $H$ coincides with the topology it inherits from $F$. The central result in this paper (Theorem 1.1) characterizes the situations where this property of coincidence of topologies holds: it is equivalent to another extension property, namely to the fact that a certain injective morphism between two free groups $F$ and $F^{\prime}$ admits an injective continuous extension between the pro- $\mathbf{V}$ completions of $F$ and $F^{\prime}$. It turns out that in the extension-closed case, this is equivalent to the fact that $H$ and its pro-V closure have equal rank.

After the proof of the main result, we list a number of immediate consequences: for instance, it follows from our result that if $\mathbf{V}$ is extensionclosed, the continuous extension of an injective endomorphism of the free group of rank 2 is always injective. In the last section, we illustrate our result by considering a simple example of an injective morphism $\varphi: F \rightarrow F^{\prime}$ between finitely generated free groups whose continuous extension $\hat{\varphi}$ to the pro- $p$ completions is not injective, and we exhibit a sequence $\left(t_{n}\right)_{n}$ of elements of $F$ whose limit points are non-trivial elements of $\operatorname{ker} \hat{\varphi}$.

\section{Injective extendability}

If $A$ is an alphabet (that is, a finite non-empty set), then $F(A)$ denotes the free group on $A$. Let $\mathbf{V}$ be a pseudovariety of finite groups: the pro- $\mathbf{V}$ topology on a group $G$ is the least topology which makes every morphism from $G$ into an element of $\mathbf{V}$ continuous. A basis of neighborhoods of 1 in this topology is given by the finite-index normal subgroups $K$ of $G$ such that $G / K \in \mathbf{V}$. 
The pro- $\mathbf{V}$ topology on $G$ is Hausdorff if and only if $G$ is residually $\mathbf{V}$. In that case, the pro- $\mathbf{V}$ topology on $G$ can be defined by an ultrametric distance function. This situation arises in particular if $G$ is a free group and $\mathbf{V}$ is a non-trivial extension-closed pseudovariety. In the sequel, we consider only pseudovarieties $\mathbf{V}$ such that free groups are residually $\mathbf{V}$.

If $G$ is a group, we denote by $\hat{G}$ the pro- $\mathbf{V}$ completion of $G$ : it is compact and totally disconnected. If $G=F(A)$, we write $\hat{F}_{\mathbf{V}}(A)$ for $\hat{G}$; this is also the free pro- $\mathbf{V}$ group on $A$. If $H \subseteq G$, we write $\mathrm{Cl}_{\mathbf{V}}(H)$ (or simply $\mathrm{Cl}(H))$ for the closure of $H$ in $G$. If $H \subseteq \hat{G}$, we write $\bar{H}$ for the closure of $H$ in $\hat{G}$. In particular, if $H \subseteq G, \bar{H}=\overline{\mathrm{Cl}(H)}$ and, if $G$ is residually $\mathbf{V}$, then $\mathrm{Cl}(H)=\bar{H} \cap G$.

We note that every morphism $\varphi: F \rightarrow F^{\prime}$ between free groups is uniformly continuous when both groups are equipped with their respective pro- $\mathbf{V}$ topologies. In particular, $\varphi$ admits a (uniquely defined) continuous extension between the pro- $\mathbf{V}$ completions, written $\hat{\varphi}: \hat{F} \rightarrow \hat{F}^{\prime}$.

For a justification of these assertions, we refer the readers, for instance, to $[\mathbf{7}]$. We now consider injective morphisms, and we state our main result.

Theorem 1.1. Let $\varphi: F(A) \rightarrow F(B)$ be an injective morphism and let $H=\varphi(F(A))$. Let $\mathbf{V}$ be a pseudovariety of groups such that free groups are residually $\mathbf{V}$. The following conditions are equivalent:

- The continuous extension of $\varphi, \hat{\varphi}: \hat{F}_{\mathbf{V}}(A) \rightarrow \hat{F}_{\mathbf{V}}(B)$ is one-to-one.

- The pro-V topology on $H$ coincides with the topology on $H$ induced by the pro- $\mathbf{V}$ topology on $F(B)$.

If, in addition, $\mathbf{V}$ is extension-closed, these properties are equivalent to:

- $H$ and $\mathrm{Cl}(H)$ have the same rank.

The proof of Theorem 1.1 follows directly from Propositions 1.4, 1.6 and 1.8 below.

\subsection{Comparing the pro- $\mathrm{V}$ topologies on a subgroup.}

We will use the following elementary remark.

Lemma 1.2. Let $\varphi: F(A) \rightarrow F(B)$ be a morphism between finitely generated free groups, let $H=\varphi(F(A))$ be the range of $\varphi$ and let $\hat{\varphi}: \hat{F}_{\mathbf{V}}(A) \rightarrow$ $\hat{F}_{\mathbf{V}}(B)$ be the continuous extension of $\varphi$ between the pro- $\mathbf{V}$ completions of $F(A)$ and $F(B)$. Then the range of $\hat{\varphi}$ is $\bar{H}$.

Proof: By continuity, we have $\hat{\varphi}\left(\hat{F}_{\mathbf{V}}(A)\right)=\hat{\varphi}(\overline{F(A)}) \subseteq \overline{\varphi(F(A))}=\bar{H}$. Of course, we also have $H \subseteq \hat{\varphi}\left(\hat{F}_{\mathbf{V}}(A)\right)$. Finally, as $\hat{F}_{\mathbf{V}}(A)$ is compact and $\hat{\varphi}$ is continuous, the group $\hat{\varphi}\left(\hat{F}_{\mathbf{V}}(A)\right)$ is closed, so $\hat{\varphi}\left(\hat{F}_{\mathbf{V}}(A)\right)=\bar{H}$. 
Next we consider the following property of a finitely generated subgroup $H$ of a free group $F(B)$ :

Property Coinc(V). The pro-V topology on $H$ coincides with the topology on $H$ induced by the pro- $\mathbf{V}$ topology on $F(B)$.

This property translates as follows.

Lemma 1.3. Let $H$ be a finitely generated subgroup of a free group $F(B)$, let $\imath: H \rightarrow F(B)$ be the natural injection of $H$ into $F(B)$, and let $\hat{\imath}: \hat{H} \rightarrow \hat{F}_{\mathbf{V}}(B)$ be the continuous extension of $\imath$ between the pro-V completions of $H$ and $F(B)$. Then $H$ has Property $\operatorname{Coinc}(\mathbf{V})$ if and only if $\hat{\imath}$ is injective. In particular, $\bar{H}$ is homeomorphic to $\hat{H}$.

Proof: This is immediate once we observe that $\hat{\imath}$ has range $\bar{H}$ (by Lemma 1.2).

We are now ready to prove the first equivalence in Theorem 1.1.

Proposition 1.4. Let $\mathbf{V}$ be a pseudovariety of groups such that free groups are residually $\mathbf{V}$. Let $\varphi, \hat{\varphi}$ and $H$ be as in the statement of Theorem 1.1. Then $\hat{\varphi}$ is injective if and only if $H$ has Property $\operatorname{Coinc}(\mathbf{V})$.

Proof: Let $\psi: F(A) \rightarrow H$ be the restriction of $\varphi$ to an isomorphism between $F(A)$ and $H$, and let $\hat{\psi}: \hat{F}_{\mathbf{V}}(A) \rightarrow \hat{H}$ be the continuous extension of $\psi$. As $\psi$ is an isomorphism, $\hat{\psi}$ is a homeomorphism.

Let $\imath: H \rightarrow F(B)$ and $\hat{\imath}: \hat{H} \rightarrow \hat{F}_{\mathbf{V}}(B)$ be as in Lemma 1.3. We observe that $\varphi=\imath \circ \psi$, so that $\hat{\varphi}=\hat{\imath} \circ \hat{\psi}$. As $\hat{\psi}$ is a homeomorphism, $\hat{\varphi}$ is one-to-one if and only if $\hat{\imath}$ is. By Lemma 1.3, this is equivalent to $H$ having Property Coinc( $\mathbf{V})$, as we wanted to prove.

\subsection{The extension-closed case.}

The following sufficient condition for a finitely generated subgroup to have Property Coinc(V) was proved in [7, Proposition 2.17]:

Proposition 1.5. If $\mathbf{V}$ is extension-closed, then every finitely generated, closed subgroup of $F(A)$ has Property $\operatorname{Coinc}(\mathbf{V})$.

We will see that this sufficient condition is not necessary (Proposition 1.7 below). However, we can immediately use this property to prove one half of the remaining equivalence.

Proposition 1.6. Let $\mathbf{V}$ be a non-trivial extension-closed pseudovariety of groups. Let $\varphi: F(A) \rightarrow F(B)$ be a morphism between free groups, let $H=\varphi(F(A))$, and let $\hat{\varphi}$ be the continuous extension of $\varphi$ between the pro-V completions of $F(A)$ and $F(B)$. If $\varphi$ and $\hat{\varphi}$ are injective, then $H$ and $\mathrm{Cl}(H)$ have equal ranks. 
Proof: By Lemma 1.2, the range of $\hat{\varphi}$ is $\bar{H}$. Since $\hat{\varphi}$ is injective between two compact spaces, it is a homeomorphism onto its image, so $\bar{H}$ is homeomorphic to the free pro- $\mathbf{V}$ group of $\operatorname{rank}|A|=\operatorname{rank}(H)$.

On the other hand, we know from Proposition 1.5 that $\mathrm{Cl}(H)$ has Property Coinc(V). Applying Lemma 1.3 to $\mathrm{Cl}(H)$, we find that $\overline{\mathrm{Cl}(H)}$ is homeomorphic to the free pro- $\mathbf{V}$ group of $\operatorname{rank} \operatorname{rank}(\mathrm{Cl}(H))$.

But $\bar{H}=\overline{\mathrm{Cl}(H)}$, so we have proved that $\operatorname{rank}(H)=\operatorname{rank}(\mathrm{Cl}(H))$.

Before we prove the reverse implication, we show the following result, which does not require the hypothesis that $\mathbf{V}$ is extension-closed.

Proposition 1.7. Let $H$ be a finitely generated subgroup of the free group $F(A)$. If $H$ is dense in the pro- $\mathbf{V}$ topology of $F(A)$ and if $\operatorname{rank}(H)=\operatorname{rank}(F(A))$, then $H$ has Property $\operatorname{Coinc}(\mathbf{V})$.

Proof: As $H$ and $F(A)$ have the same rank, we may consider an injective endomorphism $\psi$ of $F(A)$ with range $H$. Let $\hat{\psi}: \hat{F}_{\mathbf{V}}(A) \rightarrow \hat{F}_{\mathbf{V}}(A)$ be the continuous extension of $\psi$. By Lemma 1.2, $\hat{\psi}$ is an onto endomorphism of $\hat{F}_{\mathbf{V}}(A)$. But every onto continuous endomorphism of a finitely generated profinite group is injective [5, Proposition 15.3]. So $\hat{\varphi}$ is injective: by Proposition 1.4, this implies that $H$ has Property $\operatorname{Coinc}(\mathbf{V})$.

We can now give the last element in the proof of Theorem 1.1.

Proposition 1.8. Let $H$ be a finitely generated subgroup of the free group $F(A)$. If $\mathbf{V}$ is extension-closed and $\operatorname{rank}(H)=\operatorname{rank}(\mathrm{Cl}(H))$, then $H$ has Property Coinc(V).

Proof: By Proposition 1.5, the pro-V topology on $\mathrm{Cl}(H)$ coincides with the topology on $\mathrm{Cl}(H)$ induced by the pro- $\mathbf{V}$ topology on $F(A)$. Therefore $H$ is dense in the pro- $\mathbf{V}$ topology on $\mathrm{Cl}(H)$. Now Proposition $1.7 \mathrm{im}-$ plies that the pro- $\mathbf{V}$ topology on $H$ coincides with the topology on $H$ induced by the pro- $\mathbf{V}$ topology on $\mathrm{Cl}(H)$, and this concludes the proof.

\section{Corollaries}

The following collection of remarks is immediately deduced from Theorem 1.1. Throughout this section, $\mathbf{V}$ denotes a pseudovariety of groups such that free groups are residually $\mathbf{V}, \varphi: F(A) \rightarrow F(B)$ is an injective morphism between free groups, $\hat{\varphi}: \hat{F}_{\mathbf{V}}(A) \rightarrow \hat{F}_{\mathbf{V}}(B)$ is the continuous extension of $\varphi$ between the pro- $\mathbf{V}$ completions of $F(A)$ and $F(B)$, and $H=\varphi(F(A))$.

Corollary 2.1. Whether $\hat{\varphi}$ is injective depends only on $H$, not on $\varphi$. 
If $\mathbf{V}=\mathbf{G}$, the pseudovariety of all finite groups, the pro- $\mathbf{V}$ completion of a group is called its profinite completion. It is well-known that for the pro-G topology, every finitely generated subgroup of the free group is closed [6]. As a result, we have:

Corollary 2.2. Every injective morphism between free groups of finite rank admits an injective continuous extension to the profinite completions of these groups.

Let $p$ be a prime number and let $\mathbf{G}_{p}$ be the pseudovariety of finite $p$-groups. The pro- $\mathbf{G}_{p}$ completion of a group is called its pro- $p$ completion. It is shown in [9] that if $p$ is a prime number, one can effectively compute the pro- $p$ closure of a finitely generated subgroup of the free group (see [7] for a polynomial time algorithm). It follows that:

Corollary 2.3. Given a prime number $p$, one can decide whether the continuous extension of $\varphi$ to the pro-p completions is injective.

Let $\mathbf{G}_{\mathrm{sol}}$ be the pseudovariety of finite solvable groups; the pro- $\mathbf{G}_{\mathrm{sol}}$ completion of a group is called its pro-solvable completion. It is also shown in [12] that one can compute the rank of the pro-solvable closure of a finite index subgroup:

Corollary 2.4. If $H$ has finite index, one can decide whether the continuous extension of $\varphi$ to the pro-solvable completions is injective.

For the general case however, we do not know whether one can effectively compute the rank of the pro-solvable closure of a given finitely generated subgroup (see the conclusion of $[\mathbf{7}]$ or [12] for a discussion). In particular, we do not know whether the injectivity of the continuous extension of $\varphi$ to the pro-solvable completions is decidable.

In [7], the pro- $\mathbf{V}$ topology is considered also when $\mathbf{V}$ is the pseudovariety $\mathbf{G}_{\text {nil }}$ of finite nilpotent groups, a pseudovariety which is not extensionclosed. An example is given of a finitely generated subgroup which is closed in that topology yet does not have Property Coinc(V) [7, Example 1.10]. This shows that the extension-closed assumption in Proposition 1.8 cannot be dispensed with. However, we also know $[\mathbf{7}]$ that the pro-nilpotent completion of the free group (its pro- $\mathbf{G}_{\text {nil }}$ completion) is a subdirect product of its pro- $p$ completions ( $p$ prime). Therefore we have:

Corollary 2.5. The continuous extension of $\varphi$ to the pro-nilpotent completions is injective if and only if, for each prime $p$, the continuous extension of $\varphi$ to the pro-p completions is injective. 
In view of Theorem 1.1, deciding the injectivity of the pro-nilpotent extension of $\varphi$ is equivalent to deciding whether every $p$-closure of $H$ has the same rank as $H$. But there are only finitely many subgroups of $F(A)$ of the form $\mathrm{Cl}_{p}(H)$, and they are effectively computable [7], [12]. It follows that:

Corollary 2.6. It is decidable whether the extension of $\varphi$ to the pronilpotent completions is injective.

Returning to extension-closed pseudovarieties, it is shown in $[\mathbf{9}]$ that $\operatorname{rank}(\mathrm{Cl}(H)) \leq \operatorname{rank}(H)$. As free groups and free pro-V groups of rank 1 are commutative, it follows that if $H$ has rank 1 or 2, then $\operatorname{rank}(H)=$ $\operatorname{rank}(\mathrm{Cl}(H))$. This translates into the following result.

Corollary 2.7. If $\mathbf{V}$ is extension-closed and $\varphi$ is defined on the free group of rank 1 or 2 , then $\hat{\varphi}$ is injective.

This last result leads to the following consequences. Let $\mathbf{G}$ be the pseudovariety of all finite groups, $B$ be a finite alphabet, and $u \in \hat{F}_{\mathbf{G}}(B)$. We say that a finite group $G$ satisfies the pseudo-identity $u=1$ if, for every continuous morphism $\psi: \hat{F}_{\mathbf{G}}(B) \rightarrow G$, we have $\psi(u)=1$. The class of finite groups which satisfy a given set of pseudo-identities is a pseudovariety, and every pseudovariety can be defined in this fashion (Reiterman's theorem, see [1] ).

Let $A=\{a, b\}, B$ be a finite alphabet, $\varphi: F(A) \rightarrow F(B)$ be an injective morphism (that is, $\varphi(a) \varphi(b) \neq \varphi(b) \varphi(a))$ and $\hat{\varphi}: \hat{F}_{\mathbf{G}}(A) \rightarrow$ $\hat{F}_{\mathbf{G}}(B)$ be the continuous extension of $\varphi$ to the free profinite groups over $A$ and $B$.

Corollary 2.8. Let $\mathbf{V}$ be a non-trivial extension-closed pseudovariety and let $\left(u_{i}\right)_{i \in I}$ be a collection of elements of $\hat{F}_{\mathbf{G}}(A)$. If $\mathbf{V}$ satisfies the pseudo-identities $\hat{\varphi}\left(u_{i}\right)=1$, then $\mathbf{V}$ satisfies the pseudo-identities $u_{i}=1$.

To build from this result, let us observe that, if $p$ is a prime number, it is immediate that a finite group is a $p$-group if and only if every one of its cyclic subgroup is a $p$-group. That is equivalent to saying that $\mathbf{G}_{p}$ is defined by a set of one-variable pseudo-identities. In fact, it is even the case that there exists a single element $u_{p} \in \hat{F}_{\mathbf{G}}(a)$ such that a finite group is a $p$-group if and only if it satisfies the pseudo-identity $u_{p}=1$ $\left(u_{p}\right.$ is the limit in the profinite topology of the sequence $a^{p^{n !}}$, denoted $u_{p}=a^{p^{\omega}}[\mathbf{2}$, Example 2.6(1)]).

It is also known that a finite group is nilpotent (resp. solvable) if and only if each of its 2-generated subgroups is nilpotent (resp. solvable), so that the pseudovarieties $\mathbf{G}_{\text {nil }}$ and $\mathbf{G}_{\text {sol }}$ are both defined by a set of 
2-variable pseudo-identities. In the nilpotent case, this is a result of Neumann and Taylor [8] and in the solvable case, it was proved by Thompson [11], see also Flavell [4]. In fact, it is known that there exists an element $u_{\text {nil }}(a, b)$ (resp. $\left.u_{\text {sol }}(a, b)\right)$ of $\hat{F}_{\mathbf{G}}(A)$ such that the single 2 -variable pseudo-identity $u_{\text {nil }}(a, b)=1$ defines exactly $\mathbf{G}_{\text {nil }}$, Almeida $[\mathbf{2}$, Example 2.7(1)] (resp. $u_{\text {sol }}(a, b)=1$ defines exactly $\mathbf{G}_{\text {sol }}$, Bandman et al. $[3])$.

Thus, Corollary 2.8 implies the following.

Corollary 2.9. Let $B$ be a finite alphabet, and let $x, y \in F(B)$ such that $x y \neq y x$. Let $\mathbf{V}$ be an extension-closed pseudovariety of groups.

- If $\mathbf{V}$ satisfies the pseudo-identity $x^{p^{\omega}}=1$, then $\mathbf{V}=\mathbf{G}_{p}$.

- If $\mathbf{V}$ satisfies the pseudo-identity $u_{\mathrm{nil}}(x, y)=1$, then $\mathbf{V}=\mathbf{G}_{p}$ for some prime $p$.

- If $\mathbf{V}$ satisfies the pseudo-identity $u_{\mathrm{sol}}(x, y)=1$, then $\mathbf{V}=\mathbf{G}_{\mathrm{sol}}$.

This can be rewritten in the, perhaps more readable, following form (without actually using the subtle results of the existence of a single pseudo-identity defining $\mathbf{G}_{p}, \mathbf{G}_{\text {nil }}$ or $\mathbf{G}_{\text {sol }}$ ).

Corollary 2.10. Let $B$ be a finite alphabet, and let $x, y \in F(B)$ such that $x y \neq y x$. Let $\mathbf{V}$ be an extension-closed pseudovariety of groups.

- If for every morphism $\psi: F(B) \rightarrow G$ into an element $G \in \mathbf{V}, \psi(x)$ has exponent a power of $p$ (for some fixed prime $p$ ), then $\mathbf{V}=\mathbf{G}_{p}$.

- If for every morphism $\psi: F(B) \rightarrow G$ into an element $G \in \mathbf{V}, \psi(x)$ and $\psi(y)$ generate a nilpotent subgroup of $G$, then $\mathbf{V}=\mathbf{G}_{p}$ for some prime $p$.

- If for every morphism $\psi: F(B) \rightarrow G$ into an element $G \in \mathbf{V}, \psi(x)$ and $\psi(y)$ generate a solvable subgroup of $G$, then $\mathbf{V}=\mathbf{G}_{\mathrm{sol}}$.

\section{An example}

We now give an explicit example of an injective morphism $\varphi$ between finitely generated free groups whose continuous extension $\hat{\varphi}$ between the corresponding free pro- $p$ groups is not injective, and we exhibit a sequence of words $\left(t_{n}\right)_{n}$ such that $\lim \varphi\left(t_{n}\right)=1$, yet 1 is not a limit point of $\left(t_{n}\right)_{n}$ : thus the limit points of $\left(t_{n}\right)_{n}$ are non-trivial elements of ker $\hat{\varphi}$. Put differently, this means that $p$-groups ultimately satisfy $\varphi\left(t_{n}\right)=1$, yet there exists a $p$-group that does not satisfy any of the identities $t_{n}=1$. 
Let $q$ be a fixed odd prime, let $B=\{x, y\}$, and let $H$ be the kernel of the morphism from $F(B)$ into the additive group $\mathbb{Z} / q \mathbb{Z}$ which maps letters $x$ and $y$ to 1 . Then $H$ has rank $q+1$ and if $A=\left\{a_{0}, a_{1}, \ldots, a_{q}\right\}$, then $H$ is the range of the injective morphism $\varphi: F(A) \rightarrow F(B)$ given by

$$
\begin{aligned}
& \varphi\left(a_{i}\right)=x^{i} y x^{-(i+1)}, \quad i=0, \ldots, q-2 \\
& \varphi\left(a_{q-1}\right)=x^{q-1} y \\
& \varphi\left(a_{q}\right)=x^{q} .
\end{aligned}
$$

As $F(B) / H$ is a $q$-group, $H$ is closed in the pro- $q$ topology: by Theorem 1.1, the continuous extension of $\varphi$ to the pro- $q$ completions of $F(A)$ and $F(B)$ is injective. On the other hand, one can show that for every other prime number $p, H$ is dense in the pro- $p$ topology (see [7, Section 3.1]), and by Theorem 1.1 again, the continuous extension of $\varphi$ to the pro- $p$ completions is not injective.

Let $u_{0}=x, v_{0}=y$, and for each $n \geq 0, u_{n+1}=u_{n} v_{n}$ and $v_{n+1}=$ $v_{n} u_{n}$. We now fix a prime number $p \neq q$. It is well-known that the identities $u_{n}=v_{n}$ are ultimately verified by every finite $p$-group (Engel identities, $[\mathbf{8}])$. This means that the sequence $\left(u_{n} v_{n}^{-1}\right)_{n}$ converges to 1 in the pro- $p$ topology. It is also easily verified that, for each $n \geq 0$, the word $u_{n} v_{n}^{-1}$ is reduced and lies in $H$. Thus there exists a (unique) word $t_{n} \in F(A)$ such that $\varphi\left(t_{n}\right)=u_{n} v_{n}^{-1}$ for each $n \geq 0$.

Let $S$ be the $q$-dimensional vector space over the $p$-element field $\mathbb{F}_{p}$ with basis $e_{0}, \ldots, e_{q-1}$. Let $\pi$ be the projection of $F(A)$ onto $S$ defined by $\pi\left(a_{i}\right)=e_{i}$ for $i=0, \ldots, q-1$ and $\pi\left(a_{q}\right)=0$. We prove that $\pi\left(t_{n}\right)$ is never 0 in $S$, so that the additive group $S$ (an abelian $p$-group) does not satisfy $t_{n}=1$.

We consider the morphism $\pi \circ \varphi^{-1}$ from $H$ to $S$. For each $n \geq 0$, let $s_{n}=\pi\left(t_{n}\right)=\pi \circ \varphi^{-1}\left(u_{n} v_{n}^{-1}\right)$. Then

$$
s_{n+1}=\pi \circ \varphi^{-1}\left(u_{n+1} v_{n+1}^{-1}\right)=\pi \circ \varphi^{-1}\left(u_{n} v_{n} u_{n}^{-1} v_{n}^{-1}\right) .
$$

Since $H$ is normal, $u_{n} v_{n} u_{n}^{-1} u_{n}^{-1} \in H$ and we have

$s_{n+1}=\pi \circ \varphi^{-1}\left(u_{n} v_{n} u_{n}^{-1} u_{n}^{-1}\right)+\pi \circ \varphi^{-1}\left(u_{n} v_{n}^{-1}\right)=s_{n}-\pi \circ \varphi^{-1}\left(u_{n} u_{n} v_{n}^{-1} u_{n}^{-1}\right)$.

Let $\sigma$ be the linear isomorphism of $S$ given by $\sigma\left(e_{i}\right)=e_{i+1}$ where the indices $i$ and $i+1$ are taken modulo $q$. We leave it to the reader to verify that, for each $i=0, \ldots, q-1$, we have

$$
\pi \circ \varphi^{-1}\left(x \varphi\left(a_{i}\right) x^{-1}\right)=\pi \circ \varphi^{-1}\left(y \varphi\left(a_{i}\right) y^{-1}\right)=\sigma \circ \pi\left(a_{i}\right) .
$$


It follows that for each $g \in F(A)$, we have

$$
\pi \circ \varphi^{-1}\left(x \varphi(g) x^{-1}\right)=\pi \circ \varphi^{-1}\left(y \varphi(g) y^{-1}\right)=\sigma \circ \pi(g) .
$$

Now $u_{n}$ is a positive word in $x$ and $y$ of length $2^{n}$, so we have

$$
s_{n+1}=s_{n}-\sigma^{2^{n}}\left(s_{n}\right)=\left(\mathrm{id}-\sigma^{2^{n}}\right)\left(s_{n}\right) .
$$

We observe that the linear transformation $\sigma$ of $V$ has order $q$ and a one-dimensional eigenspace associated to the eigenvalue 1 (generated by $\left.w=e_{0}+\cdots+e_{q-1}\right)$ and the supplementary hyperplane $W$ of equation $x_{0}+\cdots+x_{q-1}=0$ is stable under $\sigma$. Now we use the fact that $\sigma^{q}=\mathrm{id}$ and $q$ is odd: for each integer $n \geq 0, \sigma$ is equal to a power of $\sigma^{2^{n}}$. As 1 is not an eigenvalue of the restriction $\sigma_{\mid W}$, it cannot be an eigenvalue of any $2^{n}$-th power of $\sigma_{\mid W}$. It follows that the linear transformation id $-\sigma^{2^{n}}$ of $W$ is invertible for all $n \geq 0$. As $s_{0} \neq 0$, we find that $s_{n}=\pi\left(t_{n}\right) \neq 0$ for each $n$, as announced.

\section{References}

[1] J. AlmeidA, "Finite semigroups and universal algebra", Series in Algebra 3, World Scientific Publishing Co., Inc., River Edge, NJ, 1994.

[2] J. Almeida, Dynamics of implicit operations and tameness of pseudovarieties of groups, Trans. Amer. Math. Soc. 354(1) (2002), 387-411 (electronic).

[3] T. M. Bandman, G.-M. Greuel, F. Grunewald, B. Kunyavskit, G. Pfister and E. Plotkin, Characterizing finite solvable groups, in preparation.

[4] P. FlavelL, Finite groups in which every two elements generate a soluble subgroup, Invent. Math. 121(2) (1995), 279-285.

[5] M. D. Fried And M. Jarden, "Field arithmetic", Ergebnisse der Mathematik und ihrer Grenzgebiete (3) 11, Springer-Verlag, Berlin, 1986.

[6] M. HALL, JR., A topology for free groups and related groups, Ann. of Math. (2) 52 (1950), 127-139.

[7] S. Margolis, M. Sapir and P. Weil, Closed subgroups in pro-V topologies and the extension problem for inverse automata, Internat. J. Algebra Comput. 11(4) (2001), 405-445.

[8] B. H. Neumann and T. TaYlor, Subsemigroups of nilpotent groups, Proc. Roy. Soc. Ser. A 274 (1963), 1-4.

[9] L. Ribes And P. A. ZALesskiI, The pro- $p$ topology of a free group and algorithmic problems in semigroups, Internat. J. Algebra Comput. 4(3) (1994), 359-374. 
[10] H. Straubing and D. Thérien, Regular languages defined by generalized first-order formulas with a bounded number of bound variables, in: "STACS 2001" (Dresden), Lecture Notes in Comput. Sci. 2010, Springer, Berlin, 2001, pp. 551-562.

[11] J. G. Thompson, Nonsolvable finite groups all of whose local subgroups are solvable, Bull. Amer. Math. Soc. 74 (1968), 383-437.

[12] P. WeIL, Computing closures of finitely generated subgroups of the free group, in: "Algorithmic problems in groups and semigroups" (Lincoln, NE, 1998), Trends Math., Birkhäuser Boston, Boston, MA, 2000, pp. 289-307.

Thierry Coulbois:

Equipe Logique

Université Paris-7

2 place Jussieu

75251 Paris Cedex 05

France

and

Al-Quds University

Occupied Palestinian Territories

E-mail address: coulbois@logique.jussieu.fr

Mark Sapir:

Department of Mathematics

Vanderbilt University

Nashville, TN 37240-0001

U.S.A.

E-mail address: msapir@math.vanderbilt.edu

http://www. math. vanderbilt.edu/ msapir

Pascal Weil:

LaBRI, CNRS

351 cours de la Libération

33405 Talence Cedex

France

E-mail address: pascal.weil@labri.fr

http://www.labri.fr/Perso/ weil

Primera versió rebuda el 10 de desembre de 2002, darrera versió rebuda el 10 d'abril de 2003. 